\title{
Orientações gerais de enfermagem no pré-operatório imediato: Uma proposta de cartilha educativa para estudantes
}

\author{
General nursing guidelines in the immediate preoperative: A proposal of educational picture for \\ students
}

Lineamientos generales de enfermería en el preoperatorio inmediato: ropuesta de folleto didáctico

para estudiantes

Recebido: 09/03/2021 | Revisado: 16/03/2021 | Aceito: 20/03/2021 | Publicado: 30/03/2021

Larissa de Faria Souza Giro

ORCID: https://orcid.org/0000-0002-5574-1848

Universidade Federal do Estado do Rio de Janeiro, Brasil

E-mail: larissasouzag@edu.unirio.br

Thaís Santos Camelo

ORCID: https://orcid.org/0000-0003-1137-7674

Universidade Federal do Estado do Rio de Janeiro, Brasil

E-mail: thais.camelo@edu.unirio.br

Adrielle Buriti Ferreira Veloso

ORCID: https://orcid.org/0000-0001-8867-1447

Universidade Federal do Estado do Rio de Janeiro, Brasil

E-mail: adrielle.buritiveloso@edu.unirio.br

Júlia dos Santos Monteiro Machado

ORCID: https://orcid.org/0000-0001-9700-200X Universidade Federal do Estado do Rio de Janeiro, Brasil E-mail: juliasmmachado@edu.unirio.br

Aline Affonso Luna

ORCID: https://orcid.org/0000-0002-7648-8634 Universidade Federal do Estado do Rio de Janeiro, Brasil E-mail: aline.luna@unirio.br

Natália Chantal Magalhães da Silva

ORCID: https://orcid.org/0000-0003-1883-4313 Universidade Federal do Estado do Rio de Janeiro, Brasil E-mail: natalia.c.silva@unirio.br

\begin{abstract}
Resumo
O estudo teve como objetivo construir e validar uma cartilha educativa sobre orientações gerais de enfermagem no préoperatório imediato para estudantes. Trata-se de um estudo metodológico, com abordagem quantitativa e descritiva, o qual envolve a investigação de métodos de obtenção, organização e análise de dados. Para o alcance dos objetivos propostos, foram percorridas três etapas: revisão integrativa da literatura, construção da cartilha educativa e validação do material construído por peritos. Dos 11 peritos convidados, sete realizaram a avaliação do material educativo e integraram este estudo. A maioria considerou a cartilha educativa compreensível, com sequência lógica, de fácil assimilação e adequada ao público alvo. O conteúdo das orientações gerais de enfermagem no pré-operatório imediato se mostrou, em sua maioria, desejável e existente. Acredita-se que esta proposta favorece a utilização de metodologias ativas e amplia as possibilidades de ensino na área da enfermagem. Sugere-se a realização de pesquisas que tenham como foco as metodologias de ensino voltadas para o enfermeiro em formação. Ressalta-se, também, a necessidade de estudos futuros que avaliem o efeito da cartilha educativa, aqui apresentada, na aquisição de conhecimentos dos estudantes; assim, será possível certificar suas implicações no processo de ensino-aprendizagem.
\end{abstract}

Palavras-chave: Orientação; Cuidados Pré-Operatório; Enfermagem; Ensino.

\begin{abstract}
The study aimed to make and validate an educational booklet on general nursing guidelines in the immediate preoperative period for students. This is a methodological study, with a quantitative and descriptive approach, which involves the investigation of methods for obtaining, organizing and analyzing data. To achieve the proposed objectives, three steps were taken: integrative literature review, assemble of the educational booklet and its validation by experts. Of the 11 invited experts, seven carried out the evaluation of the educational material and integrated this study. Most considered the educational booklet to be practical, with a logical sequence, easy to assimilate and suitable for the target audience. The content of general nursing guidelines in the immediate preoperative period proved to be mostly desirable and existing. It is believed that this proposal favors the use of active methodologies and expands the teaching
\end{abstract}


possibilities in the field of nursing. It is suggested to carry out research that focuses on teaching methodologies aimed at the nurse in training. It is also emphasized the need for future studies to assess the effect of the educational booklet, presented here, on the acquisition of students' knowledge; thus, it will be possible to certify its implications in the teaching-learning process.

Keywords: Orientation; Preoperative Care; Nursing; Teaching.

\section{Resumen}

El estudio tuvo como objetivo construir y validar un folleto educativo sobre pautas generales de enfermería en el período preoperatorio inmediato para los estudiantes. Se trata de un estudio metodológico, con un enfoque cuantitativo y descriptivo, o que implica la investigación de métodos de obtención, organización y análisis de datos. Para alcanzar los objetivos propuestos se recorrieron tres pasos: revisión integradora de la literatura, construcción del cuaderno educativo y validación del material construido por expertos. De los 11 expertos invitados, siete realizaron la evaluación del material educativo e integraron este estudio. La mayoría consideró comprensible el cuaderno educativo, con una secuencia lógica, fácil de asimilar y adecuado para el público objetivo. El contenido de las guías generales de enfermería en el preoperatorio inmediato resultó ser, en su mayoría, deseable y existente. Se cree que esta propuesta favorece el uso de metodologías activas y amplía las posibilidades de la docencia en el campo de la enfermería. Se sugiere realizar una investigación que se enfoque en metodologías de enseñanza dirigidas al enfermero en formación. También se enfatiza la necesidad de estudios futuros para evaluar el efecto del folleto educativo, aquí presentado, en la adquisición de conocimientos de los estudiantes; así, se podrá certificar sus implicaciones en el proceso de enseñanza-aprendizaje. Palabras clave: Orientación; Cuidados Preoperatorios; Enfermería; Ensenãnza.

\section{Introdução}

O estudante de enfermagem lida com disciplinas distintas e, durante o seu processo de formação, deve adquirir conhecimentos nas mais diversas áreas que amparam a profissão (Silva, 2010).

Na clínica cirúrgica, mais especificamente no período pré-operatório, podem ocorrer sentimentos de ansiedade, angústia e medo, dado que o paciente - foco do cuidado será submetido a um processo invasivo e, certas vezes, desconhecido. Dessa forma, os impactos da hospitalização, e consequente intervenção cirúrgica, podem levar o paciente a desequilíbrios emocionais e psicossociais (Torezan, 2016). Logo, torna-se fundamental que o profissional em formação seja preparado para o acolhimento desse paciente, priorizando necessidades e realizando orientações de forma eficiente (Barbosa, 2014).

As orientações pré-operatórias caracterizam o preparo do paciente para o procedimento cirúrgico e têm como objetivo esclarecer dúvidas, indicar a necessidade de realização de exames e informar quanto às possíveis complicações, dentre outros (Barbosa, 2014). Tais orientações ocorrem no pré-operatório mediato, que compreende o período desde a marcação da cirurgia até 24 horas antes do procedimento cirúrgico; e, no pré-operatório imediato, que consiste nas 24 horas que antecedem a cirurgia (Grittem, 2006).

Geralmente, é durante o pré-operatório imediato que ocorre a hospitalização para o preparo cirúrgico, o que pode desencadear sinais e sintomas relacionados ao estresse e à ansiedade. Há uma quebra na rotina do paciente, que agora ocupa um ambiente não familiar e tem que lidar com a aproximação de sua cirurgia. Isto posto, o pré-operatório imediato surge como uma possibilidade de fortalecer o vínculo entre profissional-paciente, esclarecer dúvidas quanto ao procedimento, fornecer orientações e validar a compreensão do paciente acerca da cirurgia (Christóforo, 2009).

Tendo em vista que as orientações de enfermagem, quando realizadas de maneira eficaz, podem auxiliar na redução da ansiedade e estimular as respostas psicológicas ao estresse antes e depois da cirurgia, é fundamental que o estudante de enfermagem tenha contato com estratégias que facilitem a aquisição e consolidação do conhecimento na área. Ademais, ao orientar, tanto o enfermeiro quanto o profissional em formação devem se atentar às necessidades cognitivas dos pacientes, respeitando o grau de instrução de cada um, dado que quanto maior o entendimento, menor o nível de ansiedade e estresse neste período (Barbosa, 2014).

Nesse sentido, a utilização de metodologias ativas no processo de ensino-aprendizagem favorece a formação ampliada dos futuros profissionais. Por se basearem em situações-problema e levarem em consideração o conhecimento e a experiência 
prévia do estudante, são eficazes na promoção do senso crítico e na valorização da autonomia (Borges, 2014).

Na enfermagem, é crescente a utilização de tais ferramentas didáticas, a exemplo da sala de aula invertida, estudos de casos, jogos, dramatizações, simulações, vídeos, aplicativos, livretos, folders e cartilhas educativas (Alvarez, 2018).

Dentre esses, destaca-se a cartilha educativa, que pode ser considerada uma ferramenta simples, lúdica e acessível, permitindo diferentes abordagens (Alfonsin, 2011). Apesar de, comumente, seu uso estar relacionado à orientação daqueles que requerem cuidados, estudos envolvendo a cartilha como recurso didático a estudantes em formação podem ser apontados (Santos et al., 2016; Castelblanco et al., 2019).

Isto posto, e diante das dificuldades encontradas pelas pesquisadoras - após a conclusão do curso de graduação em enfermagem e enquanto alunas de um curso de pós-graduação em clínica médica e cirúrgica - em assimilar o conhecimento adquirido em torno da temática envolvendo as orientações de enfermagem no pré-operatório imediato, surgiu o desejo de aprofundar os estudos em ferramentas que facilitem a aquisição e a consolidação de informações na área.

Deste modo, este estudo teve como objetivo construir e validar uma cartilha educativa sobre orientações gerais de enfermagem no pré-operatório imediato para estudantes.

\section{Metodologia}

Trata-se de um estudo metodológico, com abordagem quantitativa e descritiva, o qual envolve a investigação de métodos de obtenção, organização e análise de dados. Este, compreende a elaboração, validação e avaliação de instrumentos, através da descrição dos passos executados a cada etapa concluída (Polit; Beck, 2019).

Segundo Pereira, Shitsuka, Parreira e Shitsuka (2018), o trabalho com metodologias ativas de aprendizagem constitui uma forma de buscar melhorias nos processos educacionais, e permitem ao docente a realização de pesquisas e reflexões para verificar quais estratégias estão funcionando melhor. Neste contexto, o estudo foi desenvolvido durante o período de julho de 2020 a fevereiro de 2021, tendo como público alvo estudantes de enfermagem.

Para o alcance dos objetivos propostos, foram percorridas três etapas: revisão integrativa da literatura, construção da cartilha educativa e validação do material construído por peritos.

$\mathrm{Na}$ primeira etapa, revisão integrativa, foi formulada uma questão norteadora para a pesquisa, seguida do estabelecimento de critérios de elegibilidade dos achados, definição das informações a serem extraídas, avaliação dos estudos incluídos na revisão e interpretação dos resultados (Whittemore, Knafl 2005).

Nesse sentido, formulou-se como questão norteadora: "Quais as orientações gerais de enfermagem no pré-operatório imediato?". Os critérios de elegibilidade adotados foram: artigos disponíveis em texto completo, publicados em português, inglês ou espanhol, nos últimos dez anos. A busca foi realizada durante o período de julho a agosto de 2020, nas bases de dados da Literatura Latino-Americana em Ciências de Saúde (LILACS), Scientific Electronic Library Online (SCIELO), Medical Literature Analysis and Retrieval System Online (MEDLINE) e Base de Dados de Enfermagem (BDENF), tendo como estratégia de busca os descritores DeCS/MeSH: "Orientação" e "Cuidados Pré-operatórios" e "Enfermagem". Destaca-se que a busca e a análise dos achados foram realizadas por duas pesquisadoras e, frente a desacordos, consultada uma terceira pesquisadora.

A segunda etapa do estudo compreendeu a construção da cartilha educativa. Para tanto, os achados da revisão foram complementados com livros, textos, manuais técnicos e com a experiência clínica das pesquisadoras. Assim, foi desenvolvido um roteiro com informações que deveriam constar na cartilha educativa. Na sequência, elaborou-se o storyboard, que compreendeu um quadro com duas colunas: uma destinada às informações textuais da cartilha e outra relacionada aos recursos ilustrativos. O desenvolvimento de imagens e a diagramação dos elementos textuais foram realizados por meio dos Programas Adobe $\circledR_{\text {Ilustrator Draw, Procreate e Canva. }}$

Na terceira e última etapa, o material construído foi encaminhado a enfermeiros peritos na área de clínica cirúrgica e/ou 
na área de metodologias ativas de ensino. Para seleção dos peritos, foram considerados os seguintes critérios adaptados de Fehring (1994): especialização ou atuação mínima de cinco anos na área. Inicialmente, foi realizado contato com três peritos e solicitado a indicação de outros possíveis peritos, constituindo-se a amostragem em rede (Polit; Beck, 2019). Todos receberam o convite para participação na pesquisa através de um email, que continha em seu corpo um link para acesso ao formulário eletrônico, disponibilizado pela Plataforma Google Forms ${ }^{\circledR}$. Tal formulário apresentava o termo de consentimento livre e esclarecido; o instrumento de caracterização dos peritos; a cartilha educativa; e, o instrumento de avaliação do material educativo - baseado no protocolo desenvolvido por Lopez (2006) e adaptado por Silva (2017), com tópicos relacionados à congruência do conteúdo, linguagem adotada e conteúdo das orientações gerais de enfermagem no pré-operatório imediato. Torna-se importante salientar que este estudo integra a pesquisa "Estratégias inovadoras em enfermagem: utilização no ensino e na assistência", registrada e aprovada pelo CEP da Universidade Federal do Estado do Rio de Janeiro - UNIRIO, sob parecer 3.764.010.

\section{Resultados}

Na revisão integrativa da literatura, foram identificados 194 artigos. Com a aplicação dos critérios de elegibilidade, 29 artigos foram incluídos, sendo 15 da LILACS, 10 da MEDLINE e quatro da BDENF. Após a análise seletiva, 29 artigos foram incluídos e, após a análise crítica, 6 artigos compuseram a revisão.

As orientações gerais de enfermagem no pré-operatório imediato, identificadas na revisão integrativa da literatura, são apresentadas no Quadro 1.

Quadro 1 - Orientações gerais de enfermagem no pré-operatório imediato, identificadas na revisão integrativa da literatura, Rio de Janeiro, RJ, 2021.

\begin{tabular}{|c|c|}
\hline Referência & $\begin{array}{c}\text { Orientações gerais de enfermagem no pré-operatório } \\
\text { imediato }\end{array}$ \\
\hline Perrando et al., 2011 & - Submeter-se a exames laboratoriais. \\
\hline Sampaio et al., 2013 & $\begin{array}{l}\text { - Apegar-se às crenças, eventos e pessoas que proporcionem } \\
\text { suporte espiritual. }\end{array}$ \\
\hline Sena et. al., 2017 & - Retirar esmaltes das unhas, prótese dentária, piercing e jóias. \\
\hline Sena et. al., 2017 & $\begin{array}{l}\text { - Realizar jejum, inclusive de líquidos, a partir da meia noite do } \\
\text { dia que antecede a cirurgia e suspender o uso da heparina. }\end{array}$ \\
\hline \multirow{4}{*}{$\begin{array}{l}\text { Sena; Nascimento; Maia, } 2013 \\
\text { Teixeira et al., } 2013 \\
\text { Santos; Henckmeer; Benedet, } 2011 \\
\text { Sena et. al., } 2017\end{array}$} & - Realizar a higiene no sítio operatório no dia da cirurgia. \\
\hline & - Realizar banho com solução degermante antes da cirurgia. \\
\hline & - Não lavar os cabelos durante o banho pela manhã. \\
\hline & - Colocar a camisola com a abertura nas costas. \\
\hline Sena et. al., 2017 & - Esvaziar a bexiga uma hora antes da cirurgia. \\
\hline
\end{tabular}




\begin{tabular}{|l|l|}
\hline & $\begin{array}{l}\text { - Tricotomia duas horas antes ou mais próximo da cirurgia com } \\
\text { o tricotomizador*. } \\
\text { - Apoiar o local de incisão, tossir e respirar profundamente. }\end{array}$ \\
\hline $\begin{array}{l}\text { Sena; Nascimento; Maia, 2013 } \\
\text { Teixeira et al., 2013 } \\
\text { Santos; Henckmeer; Benedet, 2011 }\end{array}$ & - Realizar exercícios respiratórios. \\
\hline
\end{tabular}

*De acordo com a Agência Nacional de Vigilância Sanitária (BRASIL, 2017) e World Health Organization (2018), recomendase que a tricotomia seja realizada somente na existência de pêlos no local de incisão cirúrgica, devendo ocorrer pouco antes da cirurgia, preferencialmente com tricotomizador elétrico (não usar lâminas) e fora da sala de cirurgia.

Fonte: Autores.

Além das orientações supracitadas, algumas ações de enfermagem no pré-operatório imediato foram apresentadas nos estudos analisados e merecem destaque, são elas: informar o paciente e seu acompanhante sobre o horário do procedimento a ser realizado; auxiliar, sempre que necessário, no alívio de sintomas relacionados à ansiedade; esclarecer dúvidas; e, validar a compreensão acerca das informações fornecidas (Sena; Nascimento; Maia, 2013; Sena et. al., 2017).

Na sequência, a partir dos achados, as informações identificadas na revisão foram complementadas com livros, textos e manuais e experiência clínica das pesquisadoras, dando origem ao roteiro da cartilha. Este, continha informações relacionadas à trâmites administrativos da intervenção cirúrgica (a exemplo de exames pré-operatórios e termo de consentimento); realização de anamnese e exame físico; horário do procedimento; retirada de próteses e adornos; uso do tricotomizador, quando necessário; necessidade de banho com aplicação de degermante no local e indicação para não lavar os cabelos; colocação da camisola com abertura nas costas; realização de exercícios respiratórios; jejum a partir de 00:00 hora do dia anterior à cirurgia; esvaziamento vesical uma hora antes do procedimento; e, esclarecimento de dúvidas, quando houver.

Finalizado o roteiro, foi elaborado o storyboard, que orientou a disposição do conteúdo em 17 páginas, com 26 imagens distintas. O desenvolvimento das imagens e a diagramação do texto foram realizados por uma pesquisadora com habilidade em recursos audiovisuais.

Assim, após os ajustes necessários, a cartilha foi encaminhada a 11 enfermeiros, peritos na área de clínica cirúrgica e/ou na área de metodologias ativas de ensino.

Dos 11 peritos convidados, sete realizaram a avaliação do material educativo e integraram este estudo. A maioria era do sexo feminino, com idade média de 37 anos ( $\mathrm{DP}=5,769)$ e média de 15 anos de formação (DP=5,802). Todos atuavam na área da enfermagem cirúrgica, sendo dois na assistência e cinco na docência. Seis dos sete peritos apresentavam especialização: quatro, em enfermagem cirúrgica; um, em saúde do adulto; e, um, em centro cirúrgico; contudo, apenas quatro referiram publicações na área. No que se refere às metodologias ativas, dois apresentavam especializações e publicações na área.

Quanto à avaliação da cartilha educativa, no que se refere à congruência de conteúdo, todos os peritos concordaram que o material permite a compreensão do tema e segue uma sequência lógica; com relação à linguagem adotada, sete apontaram que a linguagem utilizada é de fácil assimilação e adequada ao público alvo.

No que diz respeito ao conteúdo das orientações gerais de enfermagem no pré-operatório imediato, os dados são apresentados na Tabela 1 . 
Tabela 1 - Avaliação dos juízes quanto ao conteúdo das orientações gerais de enfermagem no pré-operatório imediato, Rio de Janeiro, RJ, 2021.

\begin{tabular}{|c|c|c|}
\hline $\begin{array}{c}\text { Conteúdo das orientações gerais } \\
\text { de enfermagem no pré- } \\
\text { operatório imediato }\end{array}$ & $\begin{array}{c}\text { Desejável } \\
(\mathbf{n}=7)\end{array}$ & $\begin{array}{l}\text { Existente } \\
(n=7)\end{array}$ \\
\hline $\begin{array}{l}\text { Importância das orientações de } \\
\text { enfermagem no período pré- } \\
\text { operatório }\end{array}$ & 6 & 5 \\
\hline $\begin{array}{l}\text { Definição de pré-operatório } \\
\text { imediato e imediato }\end{array}$ & 5 & 5 \\
\hline $\begin{array}{l}\text { Orientações gerais de enfermagem } \\
\text { no pré-operatório imediato: }\end{array}$ & & \\
\hline Horário da cirurgia & 6 & 5 \\
\hline $\begin{array}{l}\text { Exames realizados no pré- } \\
\text { operatório }\end{array}$ & 6 & 5 \\
\hline $\begin{array}{l}\text { Banho com clorexidina } 2 \% \text { no dia } \\
\text { da cirurgia (sem lavar os cabelos) }\end{array}$ & 7 & 4 \\
\hline $\begin{array}{l}\text { Proteção à incisão cirúrgica, após } \\
\text { o procedimento, ao tossir e } \\
\text { espirrar }\end{array}$ & 5 & 5 \\
\hline $\begin{array}{l}\text { Colocação da camisola com } \\
\text { abertura para as costas }\end{array}$ & 7 & 4 \\
\hline $\begin{array}{l}\text { Retirada de peças íntimas, } \\
\text { esmalte, adornos e próteses }\end{array}$ & 5 & 5 \\
\hline Colocação de toucas e propés & 6 & 5 \\
\hline Esclarecimento de dúvidas & 7 & 4 \\
\hline Tricotomia & 7 & 4 \\
\hline $\begin{array}{l}\text { Checagem do termo de } \\
\text { consentimento }\end{array}$ & 6 & 5 \\
\hline $\begin{array}{l}\text { Orientação quanto ao } \\
\text { esvaziamento vesical }\end{array}$ & 6 & 5 \\
\hline Aporte emocional ao paciente & 6 & 5 \\
\hline
\end{tabular}

Fonte: Autores (2021).

Verifica-se que os tópicos "banho com clorexidina $2 \%$ no dia da cirurgia (sem lavar os cabelos)", "colocação de camisola com abertura nas costas", "esclarecimento de dúvidas" e "tricotomia", referentes às orientações gerais de enfermagem no período pré-operatório imediato, todos os peritos os consideram desejáveis. No entanto, apenas quatro peritos apontaram que tais tópicos estavam presentes na cartilha educativa.

As sugestões propostas foram analisadas pelas pesquisadoras e acatadas, quando pertinentes. Cabe destacar a recomendação para incluir na cartilha tópicos relacionados à segurança do paciente, como a utilização de pulseira de identificação e o auxílio da enfermagem durante o banho.

Após os ajustes, a versão final da cartilha educativa apresentou 17 páginas, divididas em: capa e contracapa, apresentação da personagem, relato acerca da importância das orientações gerais de enfermagem no período pré-operatório, apresentação da definição de pré-operatório mediato e imediato e apresentação das orientações gerais de enfermagem no período pré-operatório imediato e referências. A cartilha se baseia nas orientações fornecidas por uma enfermeira do setor de clínica cirúrgica - personagem: Enfermeira Ana - à uma paciente (personagem: Vera) que se encontra em pré-operatório imediato, acompanhada de sua filha. 
A Figura 1 apresenta algumas páginas da versão final da cartilha educativa.

Figura 1 - Páginas da versão final da cartilha educativa sobre orientações gerais de enfermagem no período pré-operatório imediato, Rio de Janeiro, RJ, 2021.

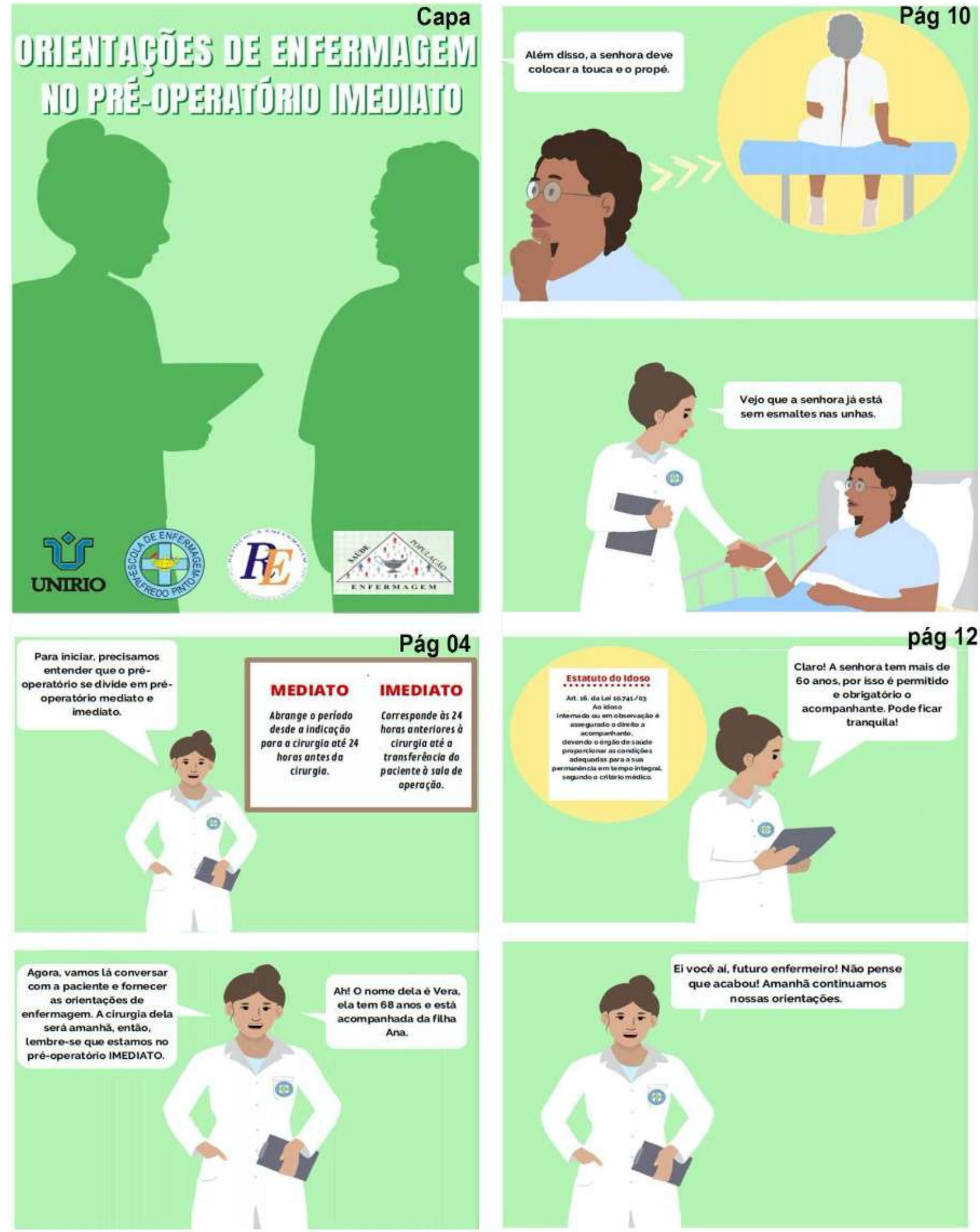

Fonte: Autores (2021). 


\section{Discussão}

No contexto atual, o processo de ensino-aprendizagem está atrelado à inovação, reforçando a importância da utilização de estratégias que favoreçam a consolidação do conhecimento de forma motivacional e prazerosa (Franco, 2013; Santo, 2013).

Nesse sentido, foram identificados quatro estudos envolvendo cartilhas educativas voltadas para estudantes do ensino superior (Sobhy, 2003; Oliveira, 2018; Carvalho, 2015; Santos, 2016); todavia, apenas um apresentava como público alvo estudantes de enfermagem (Sobhy, 2003).

Visando proporcionar o conhecimento de forma dinâmica, Sobhy e colaboradores (2003) desenvolveram uma cartilha educativa sobre o autoexame das mamas e distribuíram para estudantes de enfermagem matriculados no primeiro ano letivo de uma universidade. O material foi elaborado após a aplicação de um questionário para avaliar o nível de conhecimento dos estudantes acerca da temática. Alguns meses após o contato da cartilha, o questionário foi novamente aplicado, sendo identificada diferença significativa entre os desempenhos dos estudantes. Dessa forma, concluiu-se que o referido material contribuiu para aquisição de conhecimentos na área.

De forma semelhante, buscou-se avaliar a efetividade de uma cartilha educativa sobre o Transtorno do Déficit de Atenção/Hiperatividade (TDAH) em estudantes universitários. Antes da avaliação de seu efeito, o conteúdo da cartilha foi avaliado por três peritos com experiência profissional na área. A análise da efetividade consistiu na identificação do nível de conhecimento sobre o assunto antes e após a leitura da cartilha educativa. Os resultados evidenciaram que o contato com o material proporcionou aumento de conhecimento por parte dos estudantes (Oliveira et al., 2018).

Já um estudo desenvolvido no norte do país concentrou-se na elaboração de uma cartilha educativa com orientação postural para estudantes universitários. Esta, assim como no presente estudo, foi construída a partir de uma revisão de literatura. Contendo uma breve introdução sobre as principais alterações posturais e apresentação de posturas adequadas e inadequadas, os pesquisadores também optaram por fazer uso de recursos ilustrativos no desenvolvimento do material educativo (Carvalho et al., 2015).

No estudo desenvolvido por Santos e colaboradores (2016), foi construída uma cartilha educativa sobre a coloração de GRAM. Compreendido como um método de coloração de bactérias e por ser uma técnica usual na disciplina de microbiologia básica, julgou-se necessário realizar a construção de um material educativo que possibilitasse a aquisição de conhecimentos de forma inovadora. A construção da cartilha também partiu de uma revisão da literatura e utilizou de recursos visuais para sua apresentação. Ao ser distribuída aos estudantes do curso de farmácia, observou-se que houve compreensão quanto à relevância do tema, assim como uma maior facilidade na fixação do conteúdo.

No desenvolvimento de materiais educativos, a exemplo da cartilha, faz-se necessário que a estratégia de ensino seja disponibilizada visando o alcance dos objetivos por ela propostos. Dessa forma, é fundamental que seja construída a partir da literatura científica e experiência clínica de pesquisadores da área. Ademais, o processo de validação contribui para o aumento da credibilidade do material (Oliveira, 2014).

Ao avaliar tópicos referentes à cartilha, no presente estudo, os peritos também puderam realizar sugestões. Nesse sentido, torna-se importante salientar que apesar de um perito ter julgado pertinente a inclusão do tipo de cirurgia à qual a personagem iria se submeter, bem como orientações em relação à administração do pré-anestésico, optou-se por não inserir tais tópicos, dado que o material traz um enfoque geral sobre as orientações de enfermagem e cada cirurgia apresenta suas particularidades, da mesma forma que a administração do pré-anestésico se diferencia a partir da cirurgia e rotina de cada instituição.

Foram consideradas limitações do estudo: o baixo número de peritos na temática, e a ausência de validação junto à população alvo. 


\section{Conclusão}

Neste estudo, a maioria dos peritos considerou a cartilha educativa sobre orientações gerais de enfermagem no préoperatório imediato compreensível, com sequência lógica, de fácil assimilação e adequada ao público alvo. O conteúdo das orientações gerais de enfermagem no pré-operatório imediato se mostrou, em sua maioria, desejável e existente.

Acredita-se que esta proposta favorece a utilização de metodologias ativas e amplia as possibilidades de ensino na área da enfermagem. Nesse sentido, a cartilha educativa sobre as orientações gerais de enfermagem no pré-operatório imediato pode facilitar a consolidação do conhecimento teórico e amparar a prática do profissional em formação.

Sugere-se a realização de pesquisas que tenham como foco as metodologias de ensino voltadas para o enfermeiro em formação. Ressalta-se, também, a necessidade de estudos futuros que avaliem o efeito da cartilha educativa, aqui apresentada, na aquisição de conhecimentos dos estudantes; assim, será possível certificar suas implicações no processo de ensinoaprendizagem.

\section{Referências}

Alvarez, A. G., Girondi, J. B. R., \& Silva Knihs, N. (2018). Metodologias ativas na formação em enfermagem perioperatória. Revista SOBECC, $23(1), 1-2$.

Barbosa, A. C., de Souza Terra, F., \& de Carvalho, J. B. V. (2014). Humanização da assistência médica e de enfermagem ao paciente no perioperatório em um hospital universitário [Humanization of medical and nursing assistance to perioperative patient at a university hospital]. Revista Enfermagem UERJ, 22(5), 699704.

Barros, L. M., Gomes, F. A. D. V., Carneiro, F. N., Galindo Neto, N. M., Frota, N. M., \& Caetano, J. Á. (2020). Knowledge and attitude of candidates to gastroplasty about perioperative: randomized clinical trial. Revista Brasileira de Enfermagem, 73(6).

Brasil. (2017). Medidas de Prevenção de Infecção Relacionada à Assistência à Saúde/Agência Nacional de Vigilância Sanitária. Brasília - Anvisa.

Camacho, A. C. L. F., Abreu, L. T. D. A., Leite, B. S., Mata, A. C. D. O., Louredo, D. D. S., \& Silva, R. P. (2014). Validation of informative booklet about the elderly demented by nurses and nursing students: an observational-transversal study. Revista de Pesquisa: Cuidado é Fundamental Online, 6(1), 8-16.

Campos, C. (2017). A Therapeutic Communication as a Professional Tool in Nursing Care. PSILOGOS, 15(1), 91-101.

Carrol-Jonhnson, R. M., \& Paquete, M, (1994). Classification of nursing diagnoses: proceedings of the Tenth Conference, Philadelphia: J.B. (p. 55-62). Limppincott.

Carvalho, G. R., Nascimento, L. d. S., Sampaio, D. T., \& Lima, R. S. (2015). Elaboração de Cartilha sobre orientação postural para estudantes universitários através de atualização bibliográfica.

Castro Rosseto, K. R. de, Nunes, K. Z., Massaroni, L., \& Fioresi, M. (2016). O processo educativo do enfermeiro sob a ótica de clientes submetidos à cirurgia cardíaca. Revista Brasileira de Pesquisa em Saúde/Brazilian Journal of Health Research, 18(4), 22-29.

Christóforo, B. E. B., \& Carvalho, D. S. (2009). Cuidados de enfermagem realizados ao paciente cirúrgico no período pré-operatório. Revista da Escola de Enfermagem da USP, 43(1), 14-22.

Dalmolin, A., Girardon-Perlini, N. M. O., Coppetti, L. D. C., Rossato, G. C., Gomes, J. S., \& Silva, M. E. N. D. (2016). Vídeo educativo como recurso para educação em saúde a pessoas com colostomia e familiares. Revista gaúcha de Enfermagem, 37(SPE).

Espírito Santo, E. do, \& Da Luz, L. C. S. (2013). Didática no ensino superior: perspectivas e desafios. Saberes: Revista interdisciplinar de Filosofia e Educação, (8).

Franco, M. A. S. (2013). Didática: uma esperança para as dificuldades pedagógicas do ensino superior. Práxis Educacional, 9(15), $147-166$.

Grittem, L., Méier, M. J., \& Gaievicz, A. P. (2006). Visita pré-operatória de enfermagem: percepções dos enfermeiros de um hospital de ensino. Cogitare Enfermagem, 11(3).

Landeros López, M., \& Carvalho, E. C. D. (2006). La comunicación terapéutica durante instalación de venoclisis: uso de la simulación filmada. Revista LatinoAmericana de Enfermagem, 14(5), 658-665.

Lopes, J. D. L., Baptista, R. C. N., Domingues, T. A. M., Ohl, R. I. B., \& Barros, A. L. B. L. D. (2020). Development and validation of a video on bed baths. Revista Latino-Americana de Enfermagem, 28.

Oliveira, A. M. D., \& Soares, E. (2018). A comunicação como ferramenta educativa no pré-operatório mediato de transplante renal. Rev. pesqui. cuid. Fundam. 753-757.

Pereira, A. S.; Shitsuka, D. M.; Parreira, F. J. \& Shitsuka, R. (2018). Metodologia da pesquisa científica. UFSM. 
Pulido Castelblanco, D. P., Pulecio Rivera, C. C., \& Patiño Chaves, O. I. (2019). Validación de una cartilla pedagógica para enseñar ética de manera crítica y reflexiva en psicología. Rev. colomb. bioét, 34-51.

Rio de Janeiro: Imprensa Oficial, 2002. Estatuto do idoso: lei federal no 10.741, de 01 de outubro de 2003. Brasília, DF: Secretaria Especial dos Direitos Humanos, 2004. Ministério da saúde. http://bvsms.saude.gov.br/bvs/publicacoes/estatuto_idoso_3edicao.pdf.

Sampaio, C. E. P., Costa, T. M. N., de Araújo, D., \& Santoro, D. C. (2013). Mecanismos de enfrentamento desencadeados por pacientes em situações estressoras: cirurgia ambulatorial [Coping mechanism by surgical patients in stress situations: ambulatory surgery]. Revista Enfermagem UERJ, 21(4), 515-520.

Santos, J. D., Henckmeier, L., \& Benedet, S. A. (2011). O impacto da orientação pré-operatória na recuperação do paciente cirúrgico. Enferm. Foco (Brasília), 184-187.

Santos, N., Veiga, P., \& Andrade, R. (2011). Importância da anamnese e do exame físico para o cuidado do enfermeiro. Revista Brasileira de Enfermagem, 64(2), 355-358.

Santos, S. L. F., Barros, K. B. N. T., Prado, R. M. D. S., \& Arraes, M. L. B. D. M. (2016). Desenvolvimento de uma cartilha educativa sobre coloração de gram em microbiologia no ensino superior.

Sena, A. C. D., Nascimento, E. R. P. D., \& Maia, A. R. C. R. (2013). Prática do enfermeiro no cuidado ao paciente no pré-operatório imediato de cirurgia eletiva. Revista Gaúcha de Enfermagem, 34(3), 132-137.

Sena, A. C. de, do Nascimento, E. R. P., Maia, A. R. C. R., \& Santos, J. L. (2017). Construção coletiva de um instrumento de cuidados de enfermagem a pacientes no pré-operatório imediato. Revista Baiana de Enfermagem. Fehring. RJ.

Silva, M. G., Fernandes, J. D., Teixeira, G. A. D. S., \& Silva, R. M. D. O. (2010). Processo de formação da (o) enfermeira (o) na contemporaneidade: desafios e perspectivas. Texto \& Contexto-Enfermagem, 19(1), 176-184.

Silva, R. M. da, de Ávila Soares, R. S., Dalla Lana, L., Birrer, J. A., \& de Souza Mostardeiro, S. C. T. (2011). Percepção de pacientes com neoplasias esofágicas e estomacais sobre orientações pré-operatórias recebidas do enfermeiro. Revista de Enfermagem da UFSM, 1(3), 431-439.

Smeltzer, S. C., \& Bare, B. G. (2019). Brunner \& Suddarth, Tratado de enfermagem médico-cirúrgica (14 ed). Guanabara Koogan.

Sobhy, S. I., Shoeib, F. M., \& Rashad, W. A. (2003). Developing and testing the effectiveness of an educational booklet about breast self examination on university nursing students' knowledge and practices. The Journal of the Egyptian Public Health Association, 78(3-4), 341-359.

Teixeira, M. V., dos Reis Corrêa, A., de Fátima Silqueira, S. M., \& Carvalho, D. V. (2013). Avaliação dos resultados das orientações pré-operatórias a pacientes submetidos à cirurgia cardíaca eletiva. Revista de Enfermagem do Centro-Oeste Mineiro.

Torezan, G. (2016). Cartilha Educativa Ilustrada: orientações para acompanhantes de crianças submetidas a intervenções cirúrgicas.

Whittemore, R., \& Knafl, K. (2005). The integrative review: updated methodology. Journal of advanced nursing, 52(5), 546-553.

World Health Organization. (2018). Protocol for surgical site infection surveillance with a focus on settings with limited resources.

Zhou, H., Liu, M., Zeng, J., \& Zhu, J. (2016). Selection of nursing teaching strategies in mainland China: A questionnaire survey. Nurse education today, 39, $147-151$ 